\title{
Formation of Acetylcholine Receptor Clusters in Mammalian Sternohyoid Muscle Regenerating in the Absence of Nerves
}

\author{
Fay M. Hansen-Smith ${ }^{1}$ \\ Department of Anatomy and Cell Biology, Medical Sciences II, University of Michigan, Ann Arbor, Michigan 48109 \\ Received April 29, 1985; accepted in revised form May 23, 1986
}

\begin{abstract}
When the sternohyoid muscle from the rat is grafted, the original muscle fibers, including the membranes at the neuromuscular junction, degenerate irreversibly. New muscle fibers regenerate inside of the basal laminae remaining from the original muscle fibers. In this study rhodamine- $\alpha$-bungarotoxin and electron microscopy have been used to demonstrate that acetylcholine receptor (AchR) clusters and synaptic folds are restored to the regenerating myotubes even when innervation to the grafts is prevented. The AchR clusters and synaptic folds colocalized with acetylcholinesterease that persisted at the original synaptic basal lamina. The AchR clusters were not restored if the original innervation band was removed from the muscle at the time of grafting. Lengths of the AchR clusters were measured in animals ranging in weight from 50 to $700 \mathrm{~g}$. The lengths of clusters in the grafts were proportional to the lengths of those in the preoperative controls, suggesting that quantitative morphogenetic information persists through the period of degeneration and regeneration. However, the distribution of the AchRs within the clusters differed slightly from controls. Extrajunctional AchR clusters were present initially, but later disappeared. The sizes of these clusters were unrelated to the sizes of the junctional AchR clusters. This study demonstrates that morphogenetic cues persist within the region of the original motor and plate, possibly associated with the synaptic basal lamina. (-) 1986 Academic Press, Ine.
\end{abstract}

\section{INTRODUCTION}

It has been known for some time that mammalian skeletal muscle, like amphibian muscle, is capable of regenerating following a severe injury (Carlson, 1972, 1978). Ischemic injury or exposure to myotoxins cause the original muscle fibers to degenerate (Carlson and Gutmann, 1976). The cell membranes, including those at the neuromuscular junction, are destroyed and new muscle fibers subsequently regenerate within the original basal laminae (Hansen-Smith and Carlson, 1979; Hansen-Smith, 1983).

During embryonic and postnatal development the lengths of junctional acetylcholine receptor (J-AchR) clusters progressively increase with time (Bevan and Steinbach, 1977; Harris, 1981; Ziskind-Conheim and Bennet, 1982). Additionally, the shape of the J-AchR clusters undergoes progressive morphological changes, particularly during the early periods of development (Steinbach, 1981; Slater, 1982). These changes, which correspond to changes in the configuration of the end plate (Kelly and Zacks, 1969; Rubenstein and Kelly, 1981; Chiu and Sanes, 1984), occur subsequent to innervation. In contrast, membrane specializations resembling those of the postsynaptic region of the neuromuscular junction appear during early regeneration in rats, prior to the return of nerves to the muscle (Hansen-Smith, 1983).

\footnotetext{
${ }^{1}$ Present address: Department of Biological Sciences, Oakland University, Rochester, Mich. 48063.
}

Bader (1982) observed comparable structures in mature (40 day) regenerated soleus muscles and established that AchRs were associated with the membrane specializations, despite the absence of intact nerves at these sites. The time at which the AchRs first appeared, however, was not established. A biochemical analysis of a corresponding model of regenerating extensor digitorum longus (EDL) muscle grafts demonstrated a peak in AchR concentration 14 days after injury (Mong et al., 1982). The status of the innervation of the regenerating muscles in that study was not clear, however.

Preliminary reports from this laboratory (HansenSmith, 1982) and that of a colleague (Womble, 1982) suggested that in rats J-AchR clusters first appear at the myotube stage of regeneration, prior to innervation. Burden et al. (1979) and McMahan et al. (1984) have reported a similar phenomenon in regenerating amphibian muscle. The colocalization of AchR clusters and acetylcholinesterase (AchE)-positive sites in regenerating muscle suggests that the AchR clusters form at the original synaptic site (McMahan et al., 1978, 1984).

The process of muscle regeneration mimics muscle development in some respects. It is not clear whether the phenomenon of J-AchR cluster formation in the absence of nerves during regeneration follows a developmental sequence analagous to that in pre- and postnatal development. It was therefore of interest to determine in this study how the length and appearance of the clusters in muscle during early regeneration compared to 
those of original J-AchR clusters in muscles from rats of different ages. The results show that in contrast to developing muscles, the sizes of clusters that form in regenerating muscle is almost immediately restored to the same size as in the original muscle. However, the AchRs within the clusters are distributed somewhat differently than in the original muscle. In addition, more than one J-AchR cluster may be associated with some of the myotubes regenerating within a single original basal lamina.

\section{METHODS}

Muscle grafts. Free muscle grafts were used as models of regenerating muscle. Injections of bupivicaine in combination with grafting insured the total destruction of the original muscle fibers (Carlson and Gutmann, 1976). The sternohyoid ( $\mathrm{SH}$ ) muscles were selected for grafting because of their well-defined innervation bands. These strap muscles are easily subdivided longitudinally to give a graft of comparable thickness in animals varying widely in size. The use of thin grafts results in a fairly uniform sequence of regeneration within the grafts rather than the pronounced centripetal gradient of maturation observed when larger muscles are grafted (Hansen-Smith and Carlson, 1979). The SH muscles were grafted into the bed of the EDL muscle (Fig. 1). The EDL site was selected as a more appropriate host site than the bed of the original SH muscle because grafts in the neck region have been found to be easily ruptured by the movement of the animals (Must, 1984). The EDL site is well-protected and muscles grafted into that site are not easily detached during the normal movements of the animals. In addition, maintenance of denervation is more readily accomplished in the leg than in the neck region.

Male Sprague-Dawley rats, 50-700 g, were used for these experiments. The rats were anesthetized with ether during surgery. The superficial SH muscles were removed from their origin and insertion. The right $\mathrm{SH}$ muscle was frozen and used as a preoperative control, while the left SH muscle was used as the graft. The left SH muscle from the smaller animals were divided in half longitudinally, using an opthalmic tissue scissors. The SH of the animals larger than about $200 \mathrm{~g}$ was separated into two strips approximtely $3 \mathrm{~mm}$ in width, and the remaining portion was discarded. The strips of muscle were injected with $0.75 \%$ bupivicaine and subsequently soaked in bupivicaine for $10 \mathrm{~min}$ to cause irreversible degeneration of any muscle fibers that might survive the ischemia of grafting. The SH muscles were then rinsed in cold saline. The EDL muscles were severed from their tendonous attachments and discarded. The SH strips were then sutured into the EDL sites. Since the SH muscle has essentially no tendon, the muscles were sutured directly to the tendon of the EDL. The hindlimbs were denervated by severing and removing 1 cm of the left and right sciatic nerves at the level of the

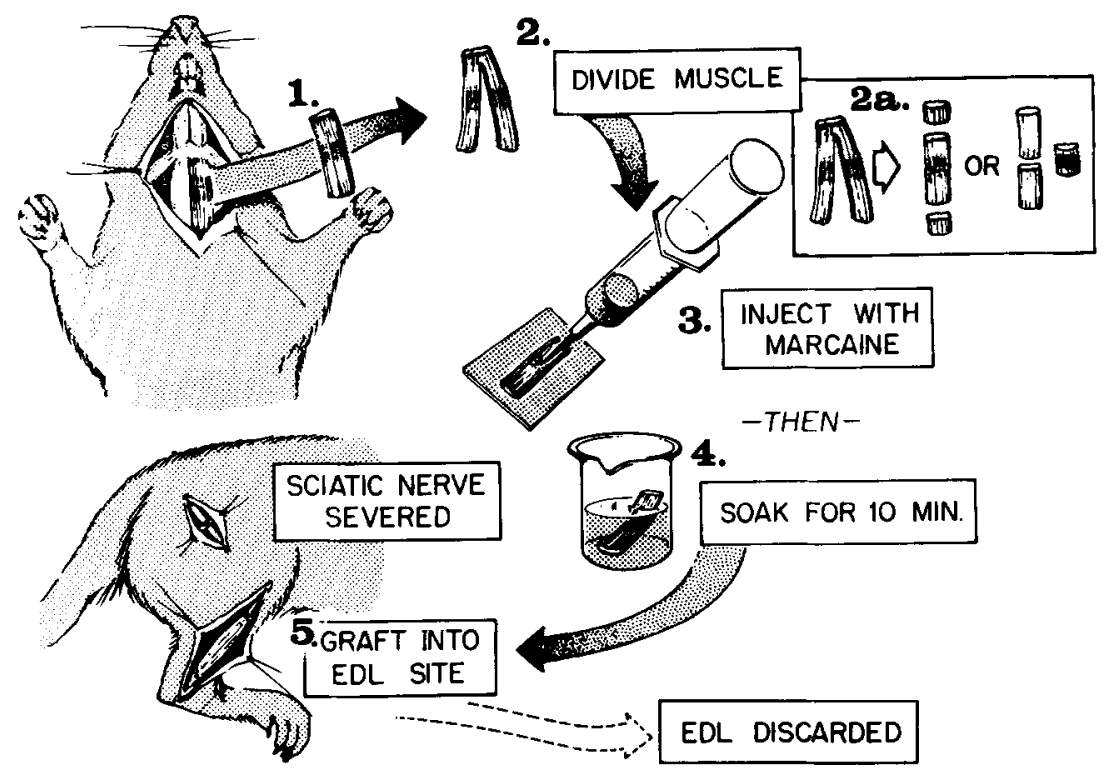

FIG. 1. Schematic depiction of grafting procedure. The sternohyoid muscles were removed from their original bed. One was frozen for use as an initial control. The remaining muscle was divided longitudinally, injected with 0.75 bupivicaine (Marcaine), and soaked in the anesthetic for $10 \mathrm{~min}$. The SH strip was then grafted into the EDL site (the EDL was removed and discarded). The sciatic nerve was severed at the level of the sciatic notch. In some experiments (2a) the innervation band or an equivalent region from a proximal of distal location, was removed and discarded. 
sciatic notch. Preliminary studies established that this procedure prevented the return of nerves to the muscles within the fourteen day period used for these experiments (unpublished observations).

The grafts were removed after 2-14 days. In six of the animals the left SH graft was removed 7 days postoperatively and the right $\mathrm{SH}$ graft was removed from the same animal 14 days postoperatively. In the remaining experiments both the left and right SH grafts were removed at the same time, the majority either 7 or 14 days postoperatively. A total of 110 grafts were used for this study.

In another experiment (six grafts) the innervation band of the muscle was surgically removed and discarded (Fig. 1). The remaining two pieces of the original muscle were then treated with bupivicaine and grafted into the EDL site. No attempt was made to suture the cut ends together. These grafts thus completely lacked the original motor end plate band. A segment of comparable size was removed and discarded from the region outside the innervation band of the control grafts. These grafts were examined after 7 or 14 days.

Preparation and analysis of frozen sections. The muscles were frozen in a mixture of isopentane $/ \mathrm{CO}_{2}$ and sectioned at a thickness of $10 \mu \mathrm{m}$. Sections were collected at five or more different levels in order to sample throughout the muscle. Rhodamine-conjugated $\alpha$-bungarotoxin was used as a ligand to detect AchRs. The method of Ravdin and Axelrod (1977) was followed for both the preparation of the toxin and for the staining of the tissue, except that the sections were prefixed with $10 \%$ Formalin to prevent folding or loss sections as the tissue was processed. This modification of the procedure had no effect on the intensity of the fluorescence. To test for the specificity of the R-BTX binding, some of the sections were exposed to $10^{-5} M$ curare, followed by incubation in a mixture of curare and R-BTX. Adjacent sections were also collected at approximately $200-\mu \mathrm{m}$ intervals and stained for AchE (Goshgarian, 1977). In a few experiments the sections were exposed to R-BTX and subsequently stained for AchE before viewing (Womble, 1983).

The frozen sections were examined on a Leitz Orthoplan microscope equipped with epifluorescent illumination. The sections were examined at a magnification of $600 \times$, using an oil immersion objective. The lengths of R-BTX binding sites in the 10- $\mu \mathrm{m}$ sections were measured with a linear microscope reticule. Measurements were made from sections at five different levels, spaced $50 \mu \mathrm{m}$ or more apart. The first $10 \mathrm{R}-\mathrm{BTX}$ sites encountered at each level were measured, giving a total of 50 measurements of J-AchR clusters for each graft or control muscle. The mean and standard deviation of the length measurements were calculated for each muscle.
Selected sections were photographed at constant magnifications $(250 \times$ or $600 \times)$, using Kodak Recording Film, with $5 \mathrm{~min}$ exposures. Longer exposure times (8-10 $\mathrm{min}$ ) were required to record the fluorescent images from tissues that had also been stained for AchE.

Significantly more interstitial cells were found in grafts than were found in controls. Many of these cells were autofluorescent, whether or not the sections were reacted with R-BTX. The autofluorescence of the interstitial cells could be distinguished from the true fluorescence of the R-BTX by a color difference (yellow vs orange-red), although the two types of fluorescence appear the same when recorded on black and white film. The autofluorescent images of the interstitial cells persisted when the specimens were examined using fluorescent filters outside the range used for rhodamine, whereas the images of the R-BTX sites were eliminated.

Electron microscopy. For electron microscopy thin strips of the muscles were fixed in $2.5 \%$ cacodylate-buffered glutaraldehyde overnight, rinsed in cacodylate buffer, postfixed in $1 \%$ cacodylate-buffered $\mathrm{OsO}_{4}$, dehydrated, and embedded. Pale gold sections were sectioned on an ultramicrotome and viewed or with uranyl acetate and lead citrate poststaining. Some of the samples were sliced into 50- $\mu \mathrm{m}$ pieces with a Farquhar-Smith tissue chopper and reacted for AchE (Karnovsky, 1964) prior to osmication and further processing. These latter specimens were generally viewed without poststaining because of a tendency for the AchE reaction product to dissolve during the poststaining procedure.

\section{RESULTS}

\section{Regeneration of the SH Muscle}

The sequence of degeneration and regeneration in the $\mathrm{SH}$ grafts was essentially the same as has been previously reported for the EDL muscle (Hansen-Smith and Carlson, 1979). All of the original muscle fibers were destroyed and the myofibrillar debris was removed by macrophages. New myotubes were present within persisting original basal laminae to the periphery of the graft by the fourth day. Regeneration was found throughout the graft by the seventh day, but there was still a slight gradient of maturity from periphery to center. By 14 days postoperatively the grafts consisted entirely of thin regenerated myofibers. AchE staining in the original innervation band persisted throughout the period of study. Electron microscopy of the original motor end plate zone revealed several regions of "presumptive synaptic folds" that were quite well developed in peripherally located regenerating muscle fibers as early as 7 days postoperatively (Fig. 2). By 14 days highly organized synaptic folds were seen that were very similar to those found in denervated, nongrafted muscle. As in comparable structures previously found in the re- 

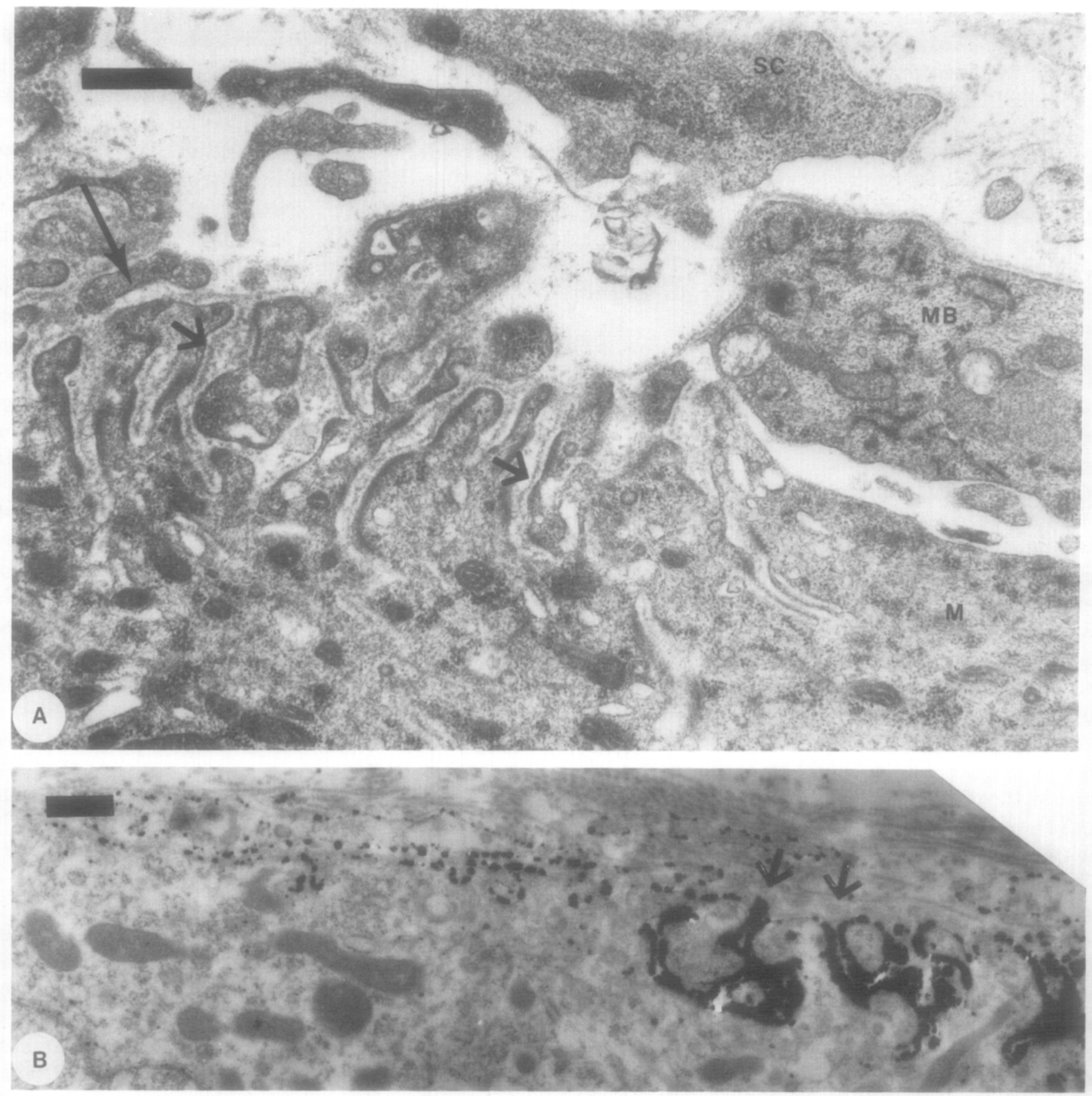

FIG. 2. Synaptic folds form in the absence of nerves in regenerating muscle. (a) Synaptic folds in sternohyoid muscle fiber 7 days after grafting are comparable to those found in a denervated muscle. Electron-dense filmentous plaques are associated with the membranes of the folds. Basal laminae is associated with most of the folds (short arrows), but is absent or amorphous in a few regions (long arrow). A second cell possibly a myoblast (MB), is enclosed within the BL of the myofiber. A portion of the Schwann cell is present (SC). The section was poststained with uranyl acetate and lead citrate. (b) Acetylcholinesterase staining reaction product is associated with synaptic folds (arrows) in a sternohyoid muscle 14 days after grafting. Occasional shallow folds are also seen in the "perijunctional" region, where the reaction product is present in lower concentration. The amount of reaction product declines in the adjacent region and is absent along most other areas of the myofiber surface. Section was viewed without poststaining. (Bar $1 \mu \mathrm{m}$ ).

generating EDL muscle (Hansen-Smith, 1983), electrondense filamentous plaques were associated with the internal aspect of the membranes at the tops of the folds. A dense AchE reaction product was associated with the basal lamina overlying and indenting into the folds (Fig. 2).

\section{$R-B T X$ Binding in Controls and Grafts}

The pattern of fluorescence in frozen sections from control SH muscles exposed to R-BTX revealed an arborized structure simialr to that of other mammalian muslces (Fig. 3, 4). In en face views the junctional (JAchR) clusters were oval or elongated, with the R-BTX 

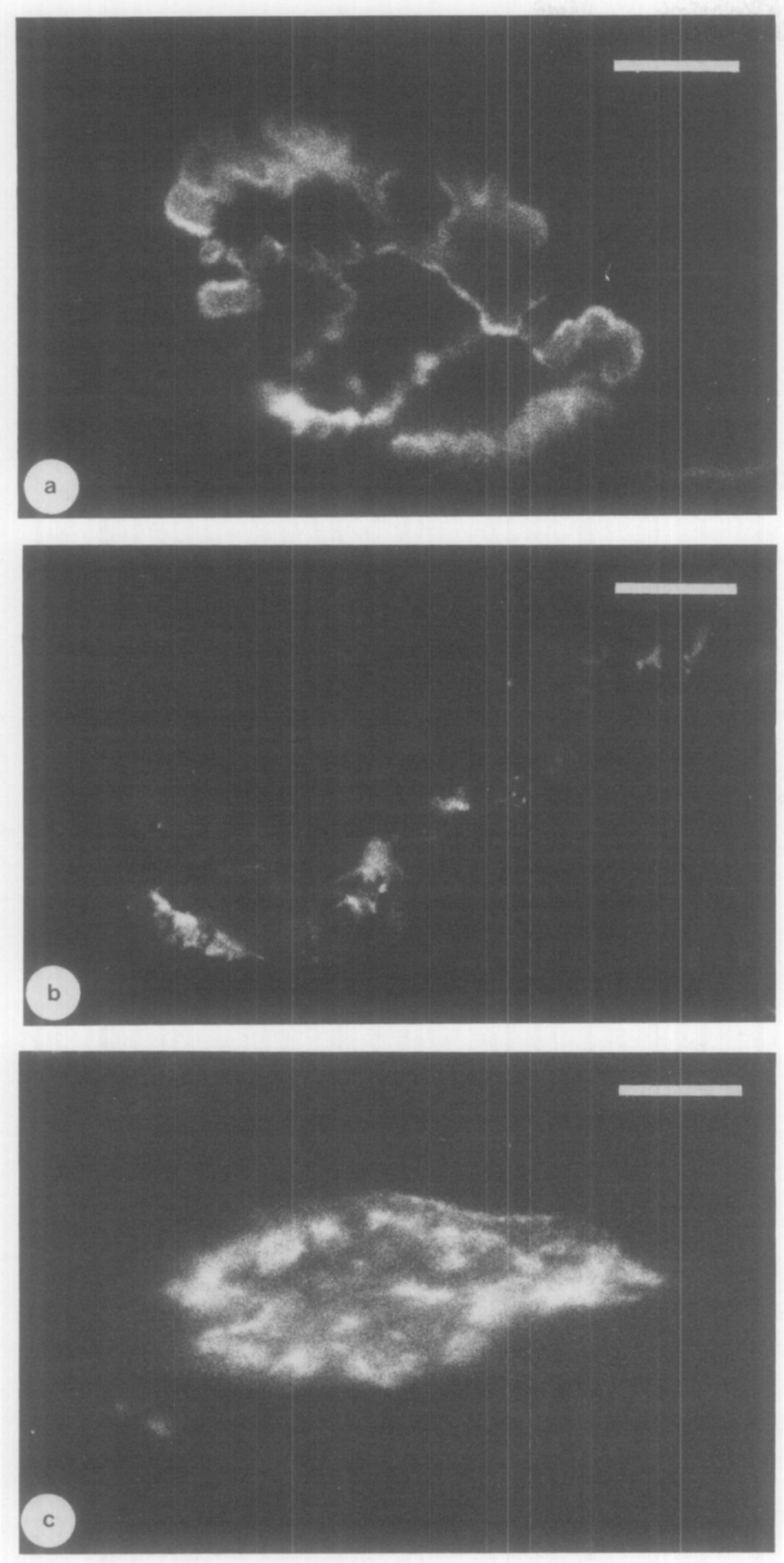

FIG. 3. En face views of R-BTX binding sites reveal J-AchR cluster in $10 \mu \mathrm{m}$-frozen sections from control and experimental sternohyoid muscles from a $300 \mathrm{~g}$ rat. (a) J-AchR clusters in control muscle reveals an oval arborized pattern of fluorescence that has precisely defined regions corresponding to the primary synaptic clefts, or gutters. (b) $\mathrm{J}$-AchR cluster from the innervation hand of a degenerating muscle 2 days after grafting. Only a few patches of R-BTX binding are observed within the junctional region. Most of the section, including the innervation band, was devoid of R-BTX binding sites. This configuration of $\mathrm{R}-\mathrm{BTX}$ binding was never observed after the degenerating myofibers had been phagocytized. (c) J-AchR cluster from the innervation band of a muscle 7 days after grafting. The R-BTX binding sites form a more stippled pattern than controls and have less precisely demarcated boundaries. Bar, $10 \mu \mathrm{m}$. staining forming spiral or branching gutters. The edges of the R-BTX gutters were precisely defined and generally stained more intensely than the more interior region of the gutter. No R-BTX binding was detectible in the spaces between the gutters or at extrajunctional locations.

Almost no R-BTX binding was detected in 2- to 3-day grafts. However, occasional sections revealed fragmented sites of R-BTX binding or very pale oval structures that were poorly defined (Fig. 3). These were located in the region of the innervation band. They were never observed after the muscle fibers had been phagocytized, suggesting that most of the original junctional AchRs were destroyed. Occasional sites of R-BTX binding were found in the junctional region as well as in the extrajunctional region of the most peripheral regenerating muscle fibers of 4-day grafts. In contrast to the few R-BTX binding sites observed in the junctional region of degenerating muscle fibers, the R-BTX formed discrete oval clusters on the surface of regenerating muscle fibers (J-AchRs). By 7 days postoperatively this pattern of binding was evident throughout the grafts. Double-labeling of the frozen sections with R-BTX and AchE revealed that the J-AchR clusters colocalized with AchE in hoth 7- and 14-day grafts (Fig. 5).

AchR clusters in 14-day grafts resembled those found at 7 days. When viewed en face, most of the clusters formed a patchy network of stippled fluorescence (Figs. $3,4)$. Both the overall pattern and the stippling of the R-BTX within the clusters distinguished the AchR clusters in the grafts from those in the controls. The patchy distribution was more pronounced in grafts from the more mature rats than in those from the 50-100 g rats.

Almost every graft examined contained examples of spatially paired AchR clusters on adjacent muscle fibers. In some cases it was obvious that the two J-AchR clusters were located on two different branches of a common parent fiber (Fig. 6). Examples of more than one J-AchR one a single muscle fiber were also observed occasionally (Fig. 6).

\section{Extrajunctional AchRS}

A second type of R-BTX binding observed was expressed in the form of small "caps" at one or more extrajunctional locations along the muscle fibers. The patches were stippled, but lacked any other morphological specialization. These will be referred to as "extrajunctional AchRs" (EJ-AchRs). The EJ-AchR clusters were numerous in 4- to 7-day grafts, but declined in number thereafter. Almost none were found in 14-day grafts. Whereas the J-AchRs corresponded in position to the AchE staining pattern of the original end plate band in adjacent sections, the EJ-AchRs were more dispersed throughout the grafts. 

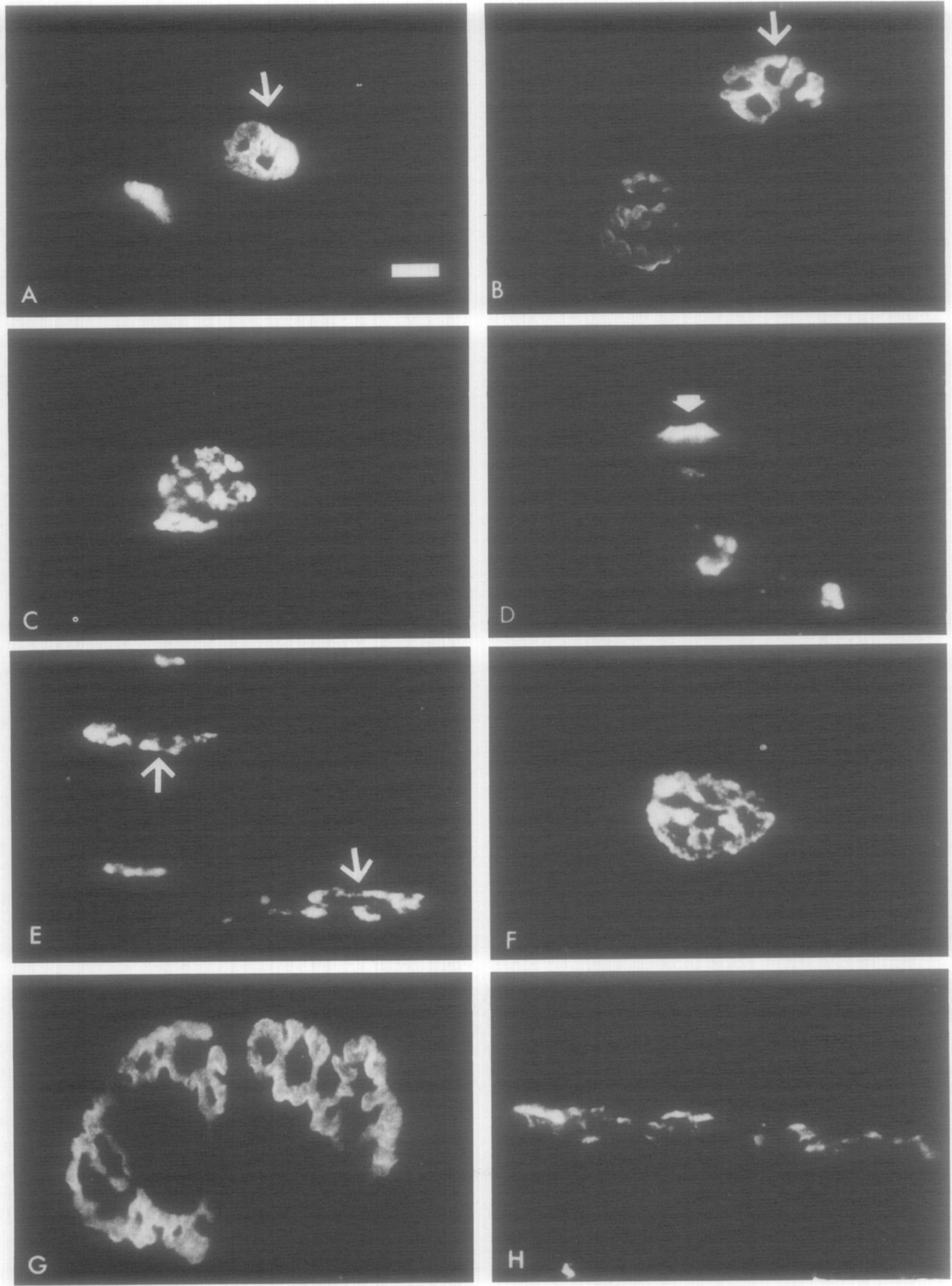

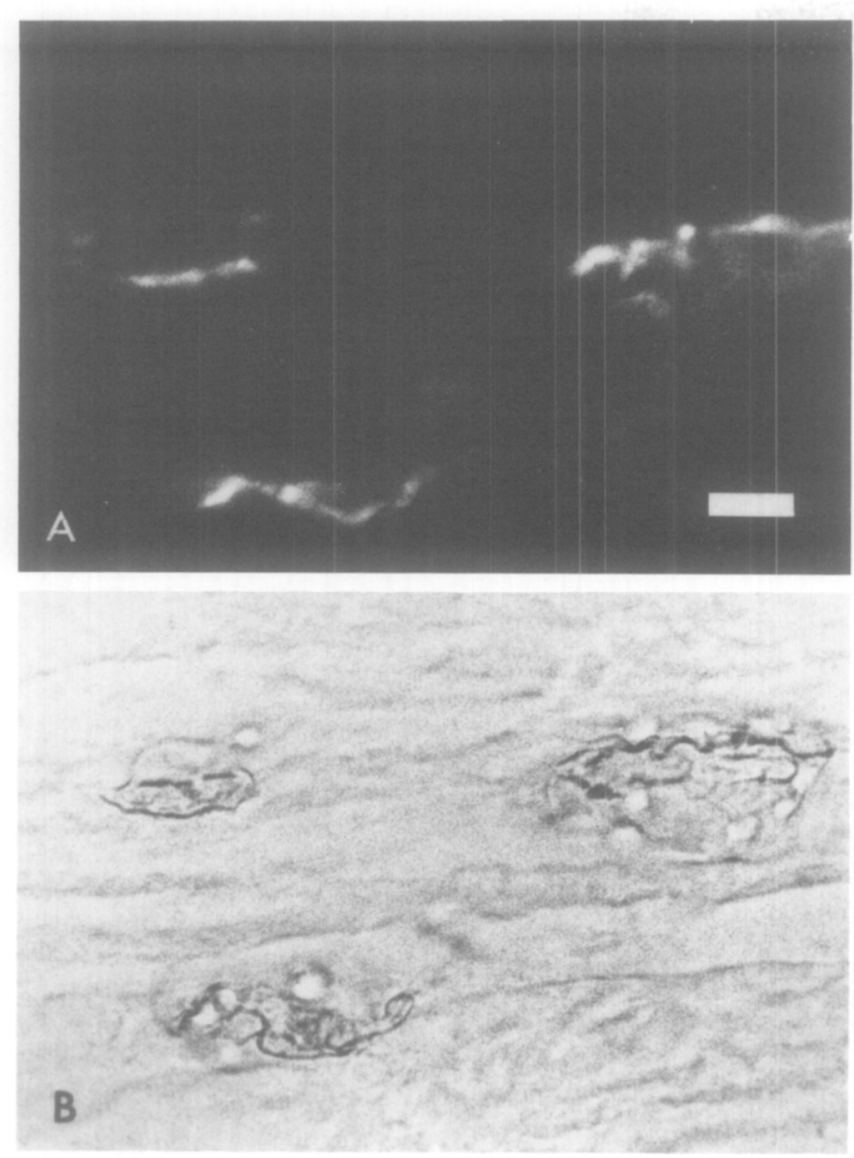

FIG. 5. The J-AchRs in regenerating muscle colocalize with acety, cholinesterase. Double-stained section from a 14-day graft was photographed by epifluorescent (a) and transmitted light (b) to show the precise alignment of three R-BTX binding sites (a) and acetylcholinesterase reaction product (b). Bar, $5 \mu \mathrm{m}$.

\section{R-BTX Binding in Grafts Lacking an Innervation Band}

Regeneration of the grafts from which the innervation band had been excised followed a time course similar to
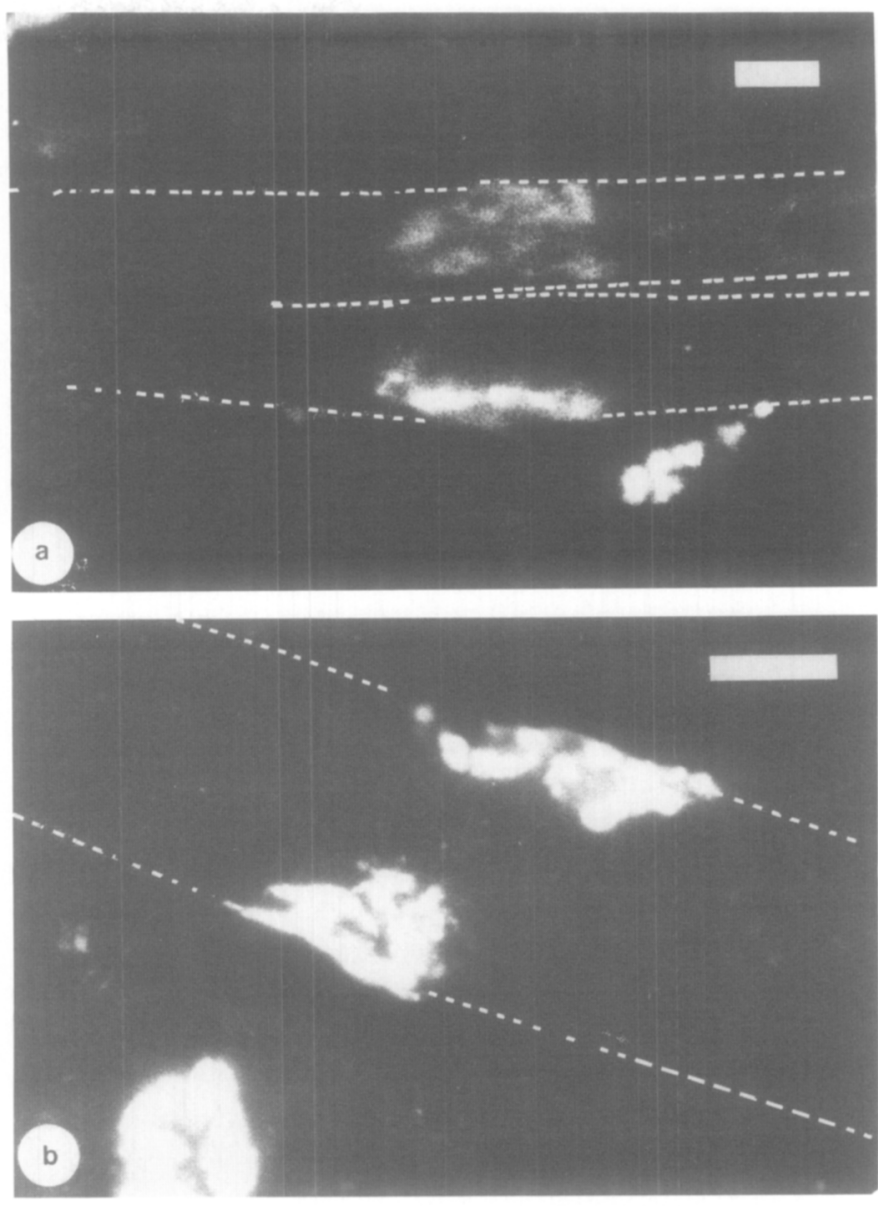

Fig. 6. Two J-AchR clusters per myofiber were occasionally observed in regenerating sternohyoid muscle. (a) The boundaries of a branching myotube in a 7-day graft are indicated by the dotted lines. One J-AchR is located on each of the two branches of the original myotube. (b) The boundaries of a myofiber in a 14-day graft are indicated by the dotted line. There was no evidence of branching in this myofiber. Two different J-AchRs are located on the opposite surfaces of the same fiber. Fluorescence found outside the dotted lines in (a) is due to interstitial cells. Bar, $10 \mu \mathrm{m}$.

FIG. 4. R-BTX binding sites at junctional and extrajunctional regions of sternohyoid muscles from rats of different size. R-BTX binding sites from age controls are shown for comparison with those of the grafted muscles in $50 \mathrm{~g}$ rats (a-f) and $700 \mathrm{~g}$ rats ( $\mathrm{g}, \mathrm{h}$ ). (a) J-AchRs in initial 50 $\mathrm{g}$ control muscle are oval and well-defined, with little arborization (arrow). A portion of an adjacent $\mathrm{J}$-AchR is evident to the left in the same plane of focus in this section. (b) J-AchRs in final control muscle 14 days after the contralateral muscle was grafted in a $50-\mathrm{g}$ rat. During this time period the J-AchR enlarged and became more complex (arrow). Portions of a second J-AchR are evident to the left, out of the plane of focus. (c) J-AchR in a grafted muscle 7 days postoperatively. An oval arborized pattern of R-BTX binding is evident, but the R-BTX is more stippled and less discretely demarcated than in either (a) or (b). The size of the JAchR resembles that of the 14-day age control in (b). (d) RBTX binding in the extrajunctional region of a 7-day graft forms a "cap" (arrow). The other fluorescent spots in the field are due to the autofluorescence of interestitial cells. (e, f) J-AchRs in 14-day grafts are shown in lateral (e) and en face (f) views. In (e) the full lengths of two J-AchRs (arrows) can be distinguished. Portions of two other J-AchRs are also evident. In (f) the arborized pattern of stippled fluorescence is evident. Both (e) and (f) are larger than in either initial (a) or 14-day (b) age controls. (g) En face view of a J-AchR from initial control muscle in a $700 \mathrm{~g}$ rat. The pattern of arborization is complex. The R-BTX binding forms a discretely defined, continuous patterns. Part of the complete length of the J-AchR cluster is out of the plane of section. (h) En face view of a J-AchR from a 14-day graft in a 700 $\mathrm{g}$ rat. The RBTX binding is stippled and either discontinuous in many regions or present in intensities too low to be visualized. The J-AchR is extremely thin and elongated in comparison to the control, partly because the myofibers in the experimental muscle are significantly thinner at this stage of regeneration than are controls. Bar, $10 \mu \mathrm{m}$. 
that of the control grafts. The only R-BTX binding observed in 7-day grafts was of the EJ-AchR type. Only a few EJ-AchRs were detected in 14-day grafts. No J-AchR clusters were observed in either 7- or 14-day grafts (data not shown).

\section{Curare Controls}

R-BTX binding at both EJ-AchR sites and at J-AchR sites was eliminated by exposure of the sections to curare (data not shown).

\section{Lengths of $J$-AchR Clusters}

The lengths of the J-AchR clusters in controls gradually increased with the size of the animals (Fig. 7). The mean length of J-AchR clusters ranged from $20.0 \pm 0.4$ $\mu \mathrm{m}$ in $50 \mathrm{~g}$ rats to $68.8 \pm 0.8 \mu \mathrm{m}$ in $700 \mathrm{~g}$ rats. Preliminary studies indicated that there was little difference between the lengths of the J-AchR clusters in 7-day and 14-day grafts (Table 1). Therefore, most of the length measurements were made on 14-day grafts because the virtual absence of EJ-AchRs simplified the measuring procedure. The lengths of the J-AchR clusters in 14-day grafts also increased with the size of the animals (29.4 $\pm 1.8 \mu \mathrm{m}-72.6 \pm 2.5 \mu \mathrm{m})$. Regression analysis of the lengths of the original vs the 14-day grafts revealed a strong correlation $(r=0.93)$ between the lengths of the original $\mathrm{J}$-AchR clusters and those of the regenerated

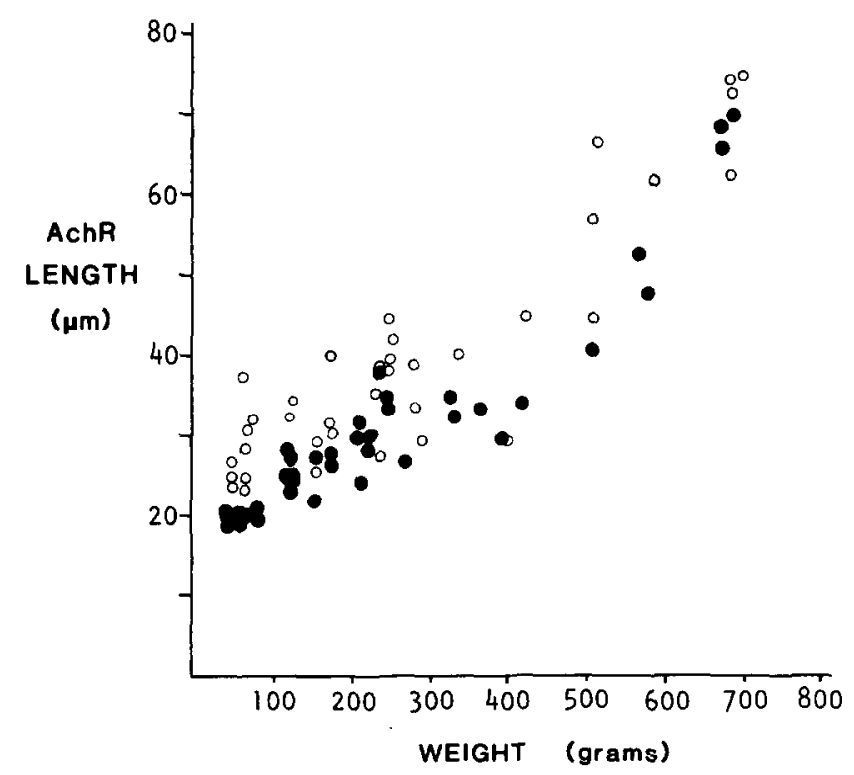

Fig. 7. The lengths of J-AchR clusters in both controls (closed circles) and 14-day grafts (open circles) were proportional to the body wieght of the rats in the range from 50 to $700 \mathrm{~g}$. Each point represents the mean of 50 length measurements taken from a total of five different sections at various levels of the muscles (see text).
TABLE 1

LENGTHS of AchR Clusters in 7- AND 14-Day STERnohyoId GraftS

\begin{tabular}{|c|c|c|c|}
\hline $\begin{array}{c}\text { Body } \\
\text { weight }\end{array}$ & $\begin{array}{c}\text { Junctional } \\
\text { AchR length }^{a} \\
\text { (7-day graft) }\end{array}$ & $\begin{array}{c}\text { Extrajunctional } \\
\text { AchR length }{ }^{a} \\
\text { (7-day graft) }\end{array}$ & $\begin{array}{c}\text { Junctional } \\
\text { AchR length }{ }^{a} \\
\text { (14-day graft) }\end{array}$ \\
\hline 70 & $23.1 \pm 0.7$ & $13.9 \pm 0.7$ & $24.8 \pm 1.2$ \\
\hline 160 & $27.8 \pm 1.5$ & $19.9 \pm 1.1$ & $30.1 \pm 0.9$ \\
\hline 170 & $29.3 \pm 1.5$ & $18.7 \pm 0.5$ & $26.6 \pm 1.1$ \\
\hline 345 & $38.8 \pm 1.5$ & $18.6 \pm 0.7$ & $41.7 \pm 1.5$ \\
\hline 400 & $42.3 \pm 3.3$ & $18.5 \pm 1.1$ & $39.9 \pm 0.8$ \\
\hline 520 & $53.7 \pm 1.9$ & $22.7 \pm 1.0$ & $57.1 \pm 3.3$ \\
\hline
\end{tabular}

${ }^{a}$ Each measurement represents the mean length $(\mu \mathrm{m}) \pm \mathrm{SEM}$ of 50 AchR clusters in a single muscle. Each of the 7- and 14-day grafts pairs were taken from the same animal, having the initial body weights indicated.

$\mathrm{J}$-AchR clusters in paired samples (Fig. 8). A comparison between the lengths of J-AchR clustes in the smallest ( $50 \mathrm{~g}, 3$ weeks and the largest $600-700 \mathrm{~g}, 8$ months) animals revealed that while the mean lengths of clusters in the largest animals were identical to controls, those of the smallest animals were actually significantly larger than controls (Table 2).

The lengths of the EJ-AchRs were only measured in a few grafts, but there was no trend toward a change in their length as the size of the animal increased (Table 1).

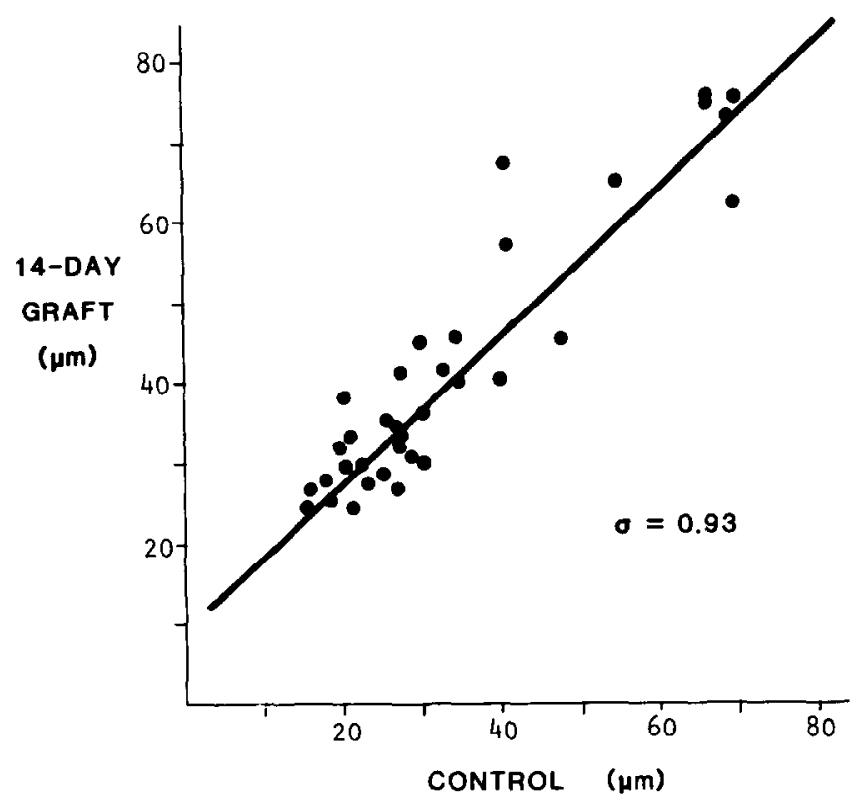

FIG. 8. The lengths of J-AchR clusters from 14-day grafted muscles are similar to those in the initial control. Each point represents the mean of 50 length measurements in initial control muscle and from 14-day grafts the same animal. Note that the lengths of J-AchRs in grafts from the smaller rats tend to be larger than those of the initial controls. The correlation coefficient for the entire sample is 0.93 . 
TABLE 2

COMPaRISON BETWEEN LengthS OF J-AchR Clusters From CONTROL MUSCI.FS AND 14-DAY STERNOHYOID GRAFTS

\begin{tabular}{|c|c|c|c|c|}
\hline $\begin{array}{l}\text { Initial } \\
\text { weight } \\
\text { (g) }\end{array}$ & Muscle & $n$ & $\begin{array}{l}\text { Length of } J- \\
\text { Ach } R \text { clusters }^{a} \\
\quad(\mu \mathrm{m})\end{array}$ & Final:initial \\
\hline 50 & Initial control & 7 & $20.0 \pm 0.4$ & \\
\hline 50 & $\begin{array}{l}\text { 14-day graft } \\
\text { Final control }\end{array}$ & 7 & $29.4 \pm 1.8^{b, c}$ & 1.47 \\
\hline 50 & for $50-\mathrm{g}$ graft & 2 & $23.2 \pm 0.7$ & \\
\hline 130 & Initial control & 4 & $25.6 \pm 0.8$ & \\
\hline 130 & 14-day graft & 4 & $31.2 \pm 1.8$ & 1.22 \\
\hline 700 & Initial control & 5 & $68.8 \pm 0.8$ & \\
\hline 700 & 14-day graft & 5 & $72.6 \pm 2.5$ & 1.05 \\
\hline
\end{tabular}

${ }^{a}$ Value represents the mean \pm SEM of the mean lengths of J-AchR clusters for $n$ muscles.

${ }^{b}$ Significantly different from initial control $(P<0.05)$.

' Significantly different from final (14-day) control $(P<0.05)$.

\section{DISCUSSION}

The original muscle fibers, including their AchRs were destroyed following grafting, but one or more new muscle fibers subsequently regenerated within the original basal laminae. The appearance of J-AchRs with the regenerating muscle at or near the site of the original neuromuscular junction indicates that morphogenetic information directing this phenomenon survives the process of degeneration of the original muscle fibers.

Several lines of evidence suggest that the AchR clusters observed in the 7- to 14-day grafts were formed at or near the site of the original neuromuscular junction: (1) J-AchR clusters were never found in grafts from which the original innervation band had been excised. (2) The AchRs invaribly aggregated in bands that corresponded in position to the AchE-reactive bands in neighboring sections. (3) Doubly stained sections revealed that the J-AchR clusters were AchE-positive. As shown in previous studies of regenerating amphibian muscle, these AchE patches identify the location of the original neuromuscular junction (Burden et al, 1979; McMahan et al., 1984). At least some of the AchE from the original neuromuscular junction is associated with the basal lamina and persists throughout the degeneration process, even when regeneration is prevented (McMahan et al., 1978). Anglister and McMahan (1985) have recently demonstrated that new AchE may be restored to the synapatic BL in the absence of nerves in regenerating amphibian muscle. Whether this is also true in mammals has not yet been determined. Previous observations in this laboratory have established, however, that in very large EDL grafts some of the original junctional AchE persists for up to 2 weeks after injury in areas of muscle that do not undergo regeneration (unpublished observations).

The process of muscle regeneration resembles that of myogenesis in that striated muscle fibers are derived from the fusion of myogenic cells. However, the two processes differ in several respects, particularly with regard to the formation of synaptic structures. During development the J-AchR clusters originate as extremely small spots that gradually enlarge (Bevan and Steinbach, 1977; Harris, 1981; Slater, 1982b; Zis-Conheim and Bennett, 1982). In striking contrast, the J-AchR clusters that formed during early regeneration are significantly larger. In fact, J-AchR clusters are proportional to the lengths of the J-AchR clusters in the same muscles prior to injury. This indicates that morphogenetic information mediating the quantitative expression of this phenomenon may be continuously modified during growth.

Although the lengths of the J-AchRs are restored to their original dimensions, the distribution of AchRs within the clusters is altered from the original configuration. The patchy or stippled appearance of the JAchR clusters in the grafts differs from the smooth contours of the arborizations in J-AchR clusters from control muscles. Similar results have been obtained in grafts of the soleus muscle (Womble, 1984). These observations further distinguish regenerating muscle from developing muscle. The morphology of J-AchR clusters and synaptic folds in developing muscle gradually evolves from a simple to a complex structure (Steinbach, 1981; Slater, 1982), whereas the J-AchR clusters and "synaptic" folds in regenerating muscle are complex as soon as they are detectible. Another diference between developing and regenerating muscles in that $\mathrm{J}$-AchR clusters in developing muscles are unstable if innervation is destroyed (Slater, 1982b), wheres the J-AchR clusters are formed and maintained for prolonged periods in the grafts in the complete absence of intact nerves (Bader, 1981; Womble, 1982; Hansen-Smith, unpublished).

Despite certain similarities in the cellular features of myoblasts and myotubes of developing and regenerating muscle, there is one important difference. Myoblasts and myotubes differentiate within a preexisting basal lamina during regeneration. This difference between regenerating muscle and developing muscle has significant implications with respect to the synaptic region of the muscle fibers. The basal lamina is a primary candidate as a regulatory factor in regenerating muscle because it is one of the few elements persisting from the original muscle fiber. The studies of McMahan et al. (1984) in regenerating amphibian muscle have essentially ruled out persisting cellular elements, i.e., Schwann cells or satellite cells, as a source of the synaptic morphogenetic information in amphibians. Several investigators have postulated that the original synaptic basal lamina di- 
rects AchR clustering, as well as other phenomena in regenerating amphibian and mammalian muscle (Sanes et al., 1978; Burden et al., 1979; Bader, 1981; McMahan et al., 1984; Glicksman and Sanes, 1983; Anglister and McMahon, 1985). Certain ubiquitous constituents of basal lamina are capable of influencing AchR clustering in vitro (Kalcheim et al., 1982; Sanes and Chiu, 1983; Vogel et al., 1983). Furthermore, synaptic BL antigens have now been characterized that either have demonstrated AchR clustering properties in vitro or precisely colocalize with AchRs (Sanes and Hall, 1979; Anderson and Fambrough, 1983; Sanes and Lawrence, 1983; Barald, el al., 1984; Bayne el al., 1984; Godfrey et al., 1984; Fallon et al., 1985). The studies lend strong support for the hypothesis that the synaptic basal lamina acts as a regulatory factor in the expression of the phenomenon of JAchR clustering in regenerating muscle.

The mechanism by which morphogenetic information is maintained and communicated in mammalian muscle during regeneration is completely unknown. The in vitro studies referred to previously have shown that solubilized factors derived from BL are capable of influencing AchR clustering. However, there are also indications that mechanical contacts also initiate AchR aggregation (Peng et al., 1981). The topographical confinement of the J-AchR clustering seems more compatable with the concept of the putative "cue" in an insoluble form within the synaptic BL.

Several observations made in the present study are of interest because they may be interpreted to suggest that information may be transmitted at points of direct contact of the regenerating muscle cells with the original BL. For example, it was observed that the J-AchRs in the grafts had a patchy and stippled appearance in comparison to the controls. This observation has also been made by Womble (1983). This might be explained by the presence of loose folds, or "ruffles" of original BL that surround the myotubes during early regeneration (Hansen-Smith and Carlson, 1979; Hansen-Smith, 1983). If the cue for AchR clustering were only transmitted at points where the myotubes contact the original synaptic BL, there would almost certainly be small gaps at the site of the "ruffles". This effect might be exaggerated in the muscle from the older rats, as the original muscle fibers would have been larger in diameter, and presumably more "ruffling" would result during degeneration. Indeed, in the present study the stippling effect and patchy distribution of AchRs was more pronounced in the J-AchRs of the larger rats. The significant lengthening of the J-AchRs in muscle from the smallest rats might also be explained by a direct contact mechanism. As the limbs of the smaller rats were rapidly elongating during the 2-week regeneration process, the BL ruffles may have been pulled out in an accordian-like fashion.
In fact, the locus of the original morphogenetic information may have been stretched, resulting in the expression of J-AchRs that were longer than the originals. Variation in the pattern and extent of synaptic BL "ruffling" at different synaptic sites in regenerating amphibian muscle might explain the variability in the density of AchRs reported by McMahan et al. (1984).

One observation in the present study is less readily explained, i.e., the finding of more than one J-AchR associated with a single original muscle fiber. Studies using antibodies to the basal lamina confirm that J-AchRs may appear in more than one site, either on a closely related site on the same myotube or on adjacent myotubes that have regenerated within a common original BL (manuscript in preparation). These observations are more difficult to explain according to a "direct contact" mechanism for the direction of AchR clustering by synaptic BL. It is possible that in a few muscle fibers the folding of the original synaptic BL is extensive enough to create two spatially different sites from which myoblasts or myotubes receive morphogenetic information. Another possible mechanism is that myoblasts or myotubes are still capable of migrating at the time they are exposed to the BL "cue," and that they move slightly from the original synaptic site before the J-AchRs form and new $\mathrm{BL}$ is deposited around the myotubes, allowing another myoblast to receive a stimulus from the same site.

The AchR clustering in the original junctional region is confined to a discrete locus in a manner that is quantitatively proportional to the length of the original neuromuscular junction. The results of the present study suggest that the morphogenetic cue(s) may be continuously modified during the growth of the original muscle. Studies in progress implicate the original nerves in the determination of the size of the J-AchRs in regenerating muscle. Some morphogenetic "cue" may be continually added as the axon terminal and J-AchR clusters elongate. The present study as well as previously reported studies (Bader, 1981; Hansen-Smith, 1983; Womble, 1983) demonstrate that nerves are not required to be present for the expression of the phenomenon of either J-AchR clustering or the formation of presumptive synaptic folds in regenerating mammalian muscle. It is possible, however, that the motor nerves impart some information to the intact muscle that facilitates the reappearance of synaptic folds and J-AchR clusters during its regeneration (Allen and Slater, 1983). Slater (1982a) has shown that in mice the lengths of J-AchR clusters are correlated with the lengths of the axon terminals during growth.

AchRs were found in extrajunctional locations during early regeneration. In contract to the increasing lengths of J-AchRs in regenerating muscle, the lengths of EJAchRs bore little relationship to the size/age of the an- 
imals. Developing muscles also have EJ-AchRs that decline in number with time. The reason for the decline in the number of EJRs is not known. However, muscle activity is known to be associated with the decline in EJAchRs during development (Ziskind-Conheim and Bennett, 1982). EJ-AchRs have been shown to contribute to $\mathrm{J}$-Ach $\mathrm{R}$ clusters in developing muscle thus possibly contributing to the decline in EJ-AchRs (Ziskind-Conheim et al., 1984). As in developing muscle, the development of spontaneous activity of the regenerating myotubes may lead to the reduction in the number of EJ-AchRs in the grafts. However, the EJ-AchRs declined in number in the grafts lacking innervation bands as well. Thus, there was no dependence on the presence of either cues within the original motor end plate band or the formation of J-AchRs for this change to occur. These studies indicate that the disappearance of EJ-AchRs cannot be attributed specifically to their migration into the junctional clusters during regeneration.

In summary, these studies support the hypothesis that the original synaptic basal lamina exerts a morphogenetic information on AchR clustering in the region of the original neuromuscular junction. The results suggest that the putative cues are confined to a very discrete locus that grows in length as the muscles mature. It is postulated that the elongation of the nerve terminals during growth may contribute to the elongation of the locus of morphogenetic information.

The author is indebted to Jill Rheinheimer and Cindy FabriciusSegal for skilled technical assistance, to John Beckerman for photography, to Bill Brudon for the illustration, to Bob Smith for assistance in the statistical analyses, and to Dr. Bruce Carlson for kindly providing laboratory space for the experiments. Mark Womble's technical expertise in the preparation and use of R-BTX and his helpful suggestions during the course of the experiments are gratefully acknowledged. Supported by NS 17017, Muscular Dystrophy Association, and Michigan Heart Association.

\section{REFERENCES}

Allen, E. G., and Slater, C. R. (1983). Acetylcholine receptor distribution at regenerating ectopic neuromuscular junctions in rat. $J$. Physiol. 343, 28.

Anderson, M. J., and Fambrough, D. M. (1983). Aggregates of acetylcholine receptors are associated with plaques of basal lamina heparan sulfate proteoglycan on the surface of skeletal muscle fibers. J. Cell. Biol. 97, 1396-1411.

ANGLISTER, L., and MCMAHAN, U. J. (1985). Basal lamina directs acetylcholinesterase accumulation at synaptic sites in regenerating muscle. J. Cell Biol. 101, 735-743.

BADER, D. (1981). Density and distribution of alpha-bungarotoxinbinding sites in postsynaptic structures of regenerated rat skeletal muscle. J. Cell Biol. 88, 338-345.

Barald, K. F., Phillips, G., Jay, J., Mizukami, I. F., Chu, D. C., Hill, J., and Pot.ACEk, L. A. (1984). A high salt extract of rat diaphragm extracellular matrix enriched in synaptic basal lamina increases the number of $\alpha$-bungarotoxin binding sites on cultured embryonic chick myotubes. Soc. Neurosci. 10, 380 .
Bayne, E. K., Anderson, M. J., Fambrough, D. M. (1984). Extracellular matrix organization in developing musele correlation with acetylcholine receptor aggregates. J. Cell Bio. 99, 1486-1501.

BEVAN, S., and STEINBACH, J. H. (1977). The distribution of $\alpha$-bungarotoxin binding sites on mammalian skeletal muscle developing in vivo. J. Physiol. 267, 195-213.

BuRden, S. J., SARGENT, P. B., and MCMAHAN, U. J. (1979). Acetylcholine receptors in regenerating muscle accumulate at original synaptic sites in the absence of the nerve. J. Cell Biol. 82, 412-425.

CARLSON, B. M. (1972). The regeneration of skeletal muscle-a review. Amer. J. Anat. 127, 119-149.

CARLSON, B. M. (1978). A review of muscle transplantation in mammals. Physiol. Bohem. 27, 387-400.

CARLSON, B. M., and GutmanN, E. (1976). Free grafting of the extensor digitorum longus muscle in the rat after marcaine pretreatment. $J$. Exp. Neurol. 53, 82-93.

Fallon, J. R., Nitkin, R. M., Reist, N. E., WALlace, B. G., and MCMAHAN, U. J. (1985). Acetylcholine receptor-aggregating factor is similar to molecules concentrated at neuromuscular junctions. Nature (Lomdon) 315, 571-574.

Glicksman, M. A., and Sanes, J. R. (1983). Differentiation of motor nerve terminals formed in the absence of muscle fibers. J. Neurocytol. 12, 661-671.

GoDfrey, E. W., Nitkin, R. M., Wallace, B. G., Rubin, L. L., MCMahaN, U. J., and MarShall, R. M. (1984). Components of torpedo electric organ and muscle that cause aggregation of acetylcholine receptors on cultured muscle cells. J. Cell Biol. 99, 615-627.

GoSHGaRIAN, H. G. (1977). A rapid silver impregnation for central and peripheral nerve fibers in paraffin and frozen sections. Exp. Neurol. 57, 296-301.

HANSEN-SMITH, F. M. (1982). Temporal sequence in reconstruction of soleplates and localization of acetylcholine receptors in regenerating sternohyoid muscles. Ant. Rec. 202, 75A.

HANSEN-SMith, F. M. (1983). Development and innervation of soleplates in the freely grafted extensor digitorum longus muscle in the rat. Ant. Rec. 207, 55-67.

HANSEN-Smith, F. M., and CARLSON, B. M. (1979). Cellular responses to free grafting of the extensor digitorum longus musele of the rat. J. Neurol. Sci. 41, 149-173.

HARRIS, A. J. (1981). Neural regulation of junctional and extrajunctional acetylcholine receptor clusters. Proc. Roy. Soc. London Ser. $B$ 293, 257-314.

KALCHEIM, C., DUKSIN, D., and VoGEL, Z. (1982). Involvement of collagen in the aggregation of acetylcholine receptors on cultured muscle cells. J. Biol. Chem. 257, 12722-12727.

KARNOVSKY, M. J. (1964). The localization of cholinesterase activity in rat cardiac muscle by electron mieroscopy. J. Cell Biol. 23, 217-232.

MatThews-Bellinger, J. A., and SalPeter, M. M. (1983). Fine structural distribution of acetylcholine receptors at developing mouse neuromuscular junctions. J. Neurosci. 3, 644-657.

McMahan, U. J., Sanes, J. R., and Marshall, L. M. (1978). Cholinesterase is associated with the basal lamina at the neuromuscular junction. Nature (London) 271, 172-174.

MCMAHAN, U. J., Slater, C. R., and Marsilall, L. M. (1984). The influence of basal lamina on the accumulation of acetylcholine receptors at synaptic sites in regenerating muscle. J. Cell Biol. 98, 1453-1473.

MonG, F. S. F., Farrach, M. C., and Martinez-Carrion, M. (1982). Pattern of acetylcholine receptor activity during regeneration of free muscle transplants of rats. J. Neurosci. Res. 8, 27-33.

MUST, R. E. (1984). "Reinnervation of Muscle Transplants." Ph.D. dissertation, University of Michigan.

Peng, H. B., Cheng, P. C., and Luther, P. W. (1981). Formation of Ach receptor clusters induced by positively charged latex beads. Nature (London) 292, 831-834. 
RAVDIN, P., and AXELROD, D. (1977). Fluorescent tetramethylrhodamine derivatives of a-bungarotoxin; preparation, separation, and characterization. Anal. Biochem. 80, 585-592.

Rubinstein, N. A., and KeLLY, A. M. (1981). Development of muscle fiber specialization in the rat hindlimb. J. Cell Biol 90, 128-144.

SANES, J. R., and CHIU, A. Y. (1983). The basal lamina of the neuromuscular junction. Cold Spring Harbor Symp. Quant. Biol 48, 667678.

SANES, J. R., and HALL, Z. W. (1979). Antibodies that bind specifically to synaptic sites on muscle fiber basal lamina. J. Cell Biol. 83, 357370.

SANES, J. R., and LAWRENCE, J. C. (1983). Activity-dependent accumulation of basal lamina by cultured rat myotubes. Dev. Biol. 97, 123-136.

SlATER, C. R. (1982a). Postnatal maturation of nerve-muscle junctions in hindlimb muscles of the mouse. Dev. Biol. 94, 11-22.

Slater, C. R. (1982b). Neural influence on the postnatal changes in acetylcholine receptor distribution at nerve-muscle junctions in the mouse. Dev. Biol 94, 23-30.
Vogei, Z., Christion, C. M., VignY, M., BaUer, H. C., SONDEREgGer, $P$, and DANIELS, M. P. (1983). Laminin induces acetylcholine receptor aggregation on cultured myotubes and enhances the receptor aggregation activity of neuronal factor. $J$. Neurosci. 31, 1058-1068.

Weinberg, C. B., Sanes, J. R., and Hald, Z. W. (1981). Formation of neuromuscular junctions in adult rats: Accumulations of acetylcholinesterase and components of synaptic basal lamina. Dev. Biol 84, 255-264.

WOMBLE, M. D. (1982). Alpha-bungarotoxin-binding site accumulation during rat skeletal muscle regeneration. Anat. Rec. 202, 207A.

Womble, M. D. (1983). "Acetylcholine Receptor Clustering and Formation of Neuromuscular Junctions in Regenerating Rat Skeletal Muscle." Ph.D. dissertation, University of Michigan.

ZISkIND-CoNHEIM, L., and BENNET, J. I. (1982). The effects of electrical inactivity and denervation on the distribution of acetylcholine receptors in developing rat muscle. Dev. Biol 90, 185-197.

Ziskind-ConhaIm, GEFFEN, L. I., and HALL, Z. W. (1984). Redistribution of acetylcholine receptors on developing rat myotubes. $J$. Neurosci. 4, 346-349. 